\title{
Estimation of boar taint in entire males on the slaughter line by examination of the genital tract
}

\author{
M. BONNEAU, Pascale RUSSEIL \\ I.N.R.A., Station de Recherches sur l'Elevage des Porcs, \\ Saint-Gilles, 35590 L'Hermitage \\ France
}

\begin{abstract}
At the present time, rearing of young boars for meat production is impossible in most countries since no satisfactory method is available for the detection of boar taint on the slaughter line. The purpose of the present study involving 451 young boars was to determine whether an examination of the genital tract could be used to estimate fat androstenone level. Coefficients of correlation between fat androstenone content and Cowper gland length ranged from 0.46 to 0.59 depending on boar groups. Multiple regressions taking into account additional criteria led only to a minor increase in the accuracy of fat androstenone estimation. The carcasses were divided into two classes "untainted " or «doubtful " using a threshold value of the estimator : "Cowper gland length», determined with the lowest possible number of errors. This threshold value varied as well as the proportion of carcasses in each of the two classes depending on the emphasis laid eiher on the errors of under-estimation (detrimental to consumers) or on the errors of over-estimation (detrimental to farmers).
\end{abstract}

\section{Relationship between the development of the muscle and its collagen content in the pig}

\author{
A. BALAND, G. MONIN \\ I.N.R.A., Station de Recherches sur la Viande. Thei.r, 63122 Ceyrat \\ France
}

The relationship between the development of the muscle and its hydroxyproline content was studied in 24 pigs of 3 genotypes with different muscle development : Large White, halothane non sensitive Pietrain and halothane sensitive Pietrain.

In our experimental conditions, no clear relationship was found between those two characters. It would be necessary to carry out further studies on a large number of breeds and to take into account other criteria pertaining to muscle development such as loin and ham percentages relative to live weight.

\author{
Effect of genotype on ham composition in female pigs \\ Comparison of Belgian Landrace, French Landrace, Pietrain \\ B.L. DUMONT, T. BOULLEAU \\ I.N.R.A., Laboratoire de Recherches sur la Viande, 78350 Jouy-cin-Josas \\ France
}

The differences in the anatomical composition of ham from female pigs were studied in the following breeds : French Landrace (FL), Belgian Landrace (BL), Pietrain (P). The 
dissection results of 16 carcasses from each breed were analysed using two methods of multidimensional analysis : centred data analysis and generalized distance of Mahalanobis's D2.

Both methods showed numerous differences between breeds as well in the anatomical tissue composition (bone, muscle, inner and outer fat percentage), as in the muscle distribution.

The FL breed exhibited a higher bone and fat percentage and a lower muscle percentage than the other breeds and the BL breed had a higher bone percentage than the P breed.

The muscle hypertrophy observed in the $\mathrm{BL}$ and $\mathrm{P}$ breeds stimulated the relative hyperdevelopment of some muscles (Adductor, Semimembranosus, Biceps femoris, Vastus lateralis) as compared to the FL breed.

The muscle distribution was not the same in the BL breed and the $\mathrm{P}$ breed where the Adductor, Gracilis and Pectineus muscles were relatively smaller. The differences observed in the muscle distribution between breeds might result from differences in the morphological traits of their bone supports.

\title{
III. - SOW PRODUCTIVITY IN FRANCE
}

\author{
Technical management of sow herds : development and results \\ Nicole COUANON, D. THOREL \\ C.E.M.A.G.R.E.F., B.P. 1312, 17, avenue de Cucillé, 35016 Rennes Codex \\ France
}

In 1981 , after 11 years of activity, the "National computerized programme for analysis of " on-the-farm sow records" is applied to $30 \mathrm{p} .100$ of the farms with more than 20 sows. It concerns 8568 herds with 777940 litters. However, the presence of the herds in the file is extremely variable.

This programme showed that a large number of criteria has been improved. In particular, the productivity has increased from 18.4 to 204 weaned piglets per sow between 1973 and 1981. Results are correlated to the size of the herds.

Examination of a smaller file involving 650 herds with reliable records and present from 1978 to 1981 showed the variability of the results for each criterion, especially in herds with the lowest or the highest performance with regard to the number of weaned piglets per sow and per year.

In 1978 and 1981 the discrepancy between the performance of the extreme herds reached 6.8 and 6.2 piglets, respectively. Even if the discrepancy is slightly smaller, the difference is still large. On an average, the herds remained in the same category in 1981, but large improvements were noticed in 15 p. 100 of the herds and large decreases in $6 \mathrm{p}$. I00) of the herds belonging to the medium category in 1978. 